\title{
Novel methods for the encapsulation of meglumine antimoniate into liposomes
}

\author{
F. Frézard ${ }^{1}$, \\ M.S.M. Michalick², \\ C.F. Soares ${ }^{2}$ and \\ C. Demicheli ${ }^{3}$
}

\author{
Departamentos de ${ }^{1}$ Fisiologia e Biofísica, and \\ 2Parasitologia, Instituto de Ciências Biológicas, and \\ ${ }^{3} \mathrm{Q}$ uímica, Instituto de Ciências Exatas, \\ Universidade Federal de M inas Gerais, Belo Horizonte, MG, Brasil
}

\section{Correspondence \\ F. Frézard \\ Departamento de Fisiologia e \\ Biofísica, ICB, UFMG \\ Av. Antônio Carlos, 6627 \\ 31270-901 Belo Horizonte, M G \\ Brasil \\ Fax: + 55-31-499-2924 \\ E-mail: frezard@mono.icb.ufmg.br \\ Research supported by $\mathrm{CNPq}$ (No. 521010/97), FAPEMIG (Nos. CEX1079/95, CBS1721/95, and CBS2418/96) and PRO NEX (No. 3075).}

Received November 25, 1999

Accepted April 10, 2000

\section{Abstract}

The antimonial drug, meglumine antimoniate, was successfully encapsulated in dehydration-rehydration vesicles and in freeze-dried empty liposomes (FDELs). High encapsulation efficiencies (from 28 to $58 \%$ ) and low weight ratios of lipids to encapsulated antimony (from 1:0.15 to 1:0.3) were achieved. These formulations, contrary to those obtained by conventional methods, can be stored as intermediate lyophilized forms and reconstituted just before use. The efficacy of FDEL-encapsulated meglumine antimoniate was evaluated in hamsters experimentally infected with Leishmania chagasi. A significant reduction of liver parasite burdens was observed in animals treated with this preparation, when compared to control animals treated with empty liposomes. In contrast, free meglumine antimoniate was found to be inefficient when administered at a comparable dose of antimony. This novel liposome-based meglumine antimoniate formulation appears to be promising as a pharmaceutical product for the treatment of visceral leishmaniasis.
In the seventies, a major advance occurred when it was found that liposomeencapsulated antimonial drugs were hundreds of times more effective than unencapsulated ones for the treatment of experimental visceral leishmaniasis in mice, hamsters and dogs $(1,2)$. Similar results were obtained with other antileishmanial agents $(3,4)$ and other vesicular systems made from nonionic surfactants, instead of phospholipids (5). This spectacular effect of liposome encapsulation was attributed to the sustained release properties of liposomes and to their natural tendency to be cleared from the circulation by the fixed macrophages of the liver, spleen
Key words

- Liposomes

- Glucantime

- Meglumine antimoniate

- Leishmaniasis

- Encapsulation and bone marrow, which are the major sites of parasite infection. It was therefore expected that liposome formulations would improve the use of antimonials, enabling a reduction in drug dose and therapy duration. However, much effort still has to be devoted to turn the experimental liposome preparations into pharmaceutical products (6).

Two different methods have been proposed so far for the encapsulation of antimonial drugs in liposomes. One method consists of the hydration of a thin film of lipids with a solution of the drug (7). The other method, known as reverse-phase evaporation procedure, involves the formation of a 
water-in-oil emulsion using the drug solution as aqueous phase followed by evaporation of the organic solvent, which results in a phase change and the formation of a vesicle suspension (8). The main advantage of the latter method, compared to the former, is that it yields higher efficiencies of drug encapsulation and higher ratios of encapsulated drug to lipid. These characteristics are important since the efficacy of liposomeencapsulated antimonials was previously shown to depend on the quantity of drug entrapped (9). These characteristics also mean that a lower quantity of lipid has to be injected in order to introduce the same quantity of antimonial, which makes the treatment safer and more economical. Nevertheless, liposomes prepared by the reverse-phase evaporation procedure may be toxic at high doses due to unavoidable residual traces of organic solvent in the final liposome formulation. Furthermore, the resulting liposome preparations can be stored only as aqueous suspensions. In this condition, however, a significant leakage of the drug occurred with time from the encapsulated aqueous phase into the continuous aqueous phase. For instance, a typical liposomal formulation prepared by the reverse-phase evaporation procedure released more than $26-48 \%$ of the originally encapsulated drug when stored for 7 weeks at $25^{\circ} \mathrm{C}(8)$. Such instability is actually unacceptable from a pharmaceutical point of view (6). Attention should also be paid to the chemical stability of the antimonial compound in such conditions. Indeed, in the specific case of meglumine antimoniate, a recent study (10) has suggested that this drug is a complex mixture of various antimony complexes, which change as a function of time and dilution.

In the present study, we have investigated some novel methods for the encapsulation of meglumine antimoniate into liposomes. In addition to the conventional thin film hydration method (7), two alternative methods were evaluated: i) a method that produces dehydration-rehydration vesicles (DRVs) (11), which involves mixing the drug solution with a suspension of pre-formed liposomes in water, freeze-drying the resulting mixture and rehydrating it in a controlled manner, and ii) a method that produces freezedried empty liposomes (FDELs) (12), which involves a controlled hydration, with the drug solution, of a lyophilized powder of empty liposomes. We report that these alternative methods display several advantages over conventional ones. They allow for the encapsulation of meglumine antimoniate with high encapsulation efficiency and they avoid stability problems during storage. The most promising formulation has been tested and found to be highly effective against experimental visceral leishmaniasis in hamsters.

Meglumine antimoniate (Glucantime) was obtained from Rhodia Brasil Ltda. (São Paulo, SP, Brasil), as an aqueous solution sealed in an ampoule. The antimony concentration was assessed at $75 \pm 7 \mathrm{~g} / \mathrm{l}$ by atomic absorption spectroscopy using a Hitachi Z8200 spectrophotometer.

The encapsulation of meglumine antimoniate into multilamellar vesicles (MLVs) was performed according to the thin film hydration procedure, as previously described (7), with the following modifications. A chloroform solution of a mixture of $72 \mathrm{mg} \mathrm{L}-\alpha$ distearoylphosphatidylcholine (DSPC; Sigma Chemical Co., St. Louis, MO, USA), $28 \mathrm{mg}$ cholesterol (CHOL, Sigma) and 10 $\mathrm{mg}$ dicetylphosphate (DCP, Sigma) was added to a round bottomed flask in the presence of 3-mm glass beads. A DSPC/CHOL/ DCP molar ratio of 5:4:1 was therefore chosen. Using a rotary evaporator, the organic solvent was removed at $50^{\circ} \mathrm{C}$ for $1 \mathrm{~h}$. MLVs were produced by hydration at $55^{\circ} \mathrm{C}$ of the thin lipid film with the solution of meglumine antimoniate $(0.8 \mathrm{ml})$ and rotation of the flask for $15 \mathrm{~min}$. The resulting suspension was diluted with $2.5 \mathrm{ml}$ phosphate-buffered saline (PBS; $0.15 \mathrm{M} \mathrm{NaCl}$ and $10 \mathrm{mM}$ phosphate, $\mathrm{pH} 7.2)$ and then centrifuged $(10,000$ 
g, $30 \mathrm{~min}, 4^{\circ} \mathrm{C}$ ) to separate unencapsulated drug from liposomes. The liposome pellet was then washed twice with $8 \mathrm{ml}$ PBS and finally resuspended in $2 \mathrm{ml}$ PBS.

The encapsulation of meglumine antimoniate into DRVs was performed as previously reported (11). Briefly, MLVs were prepared from DSPC/CHOL/DCP (molar ratio of $5: 4: 1$ ), as described above, using distilled water instead of meglumine antimoniate as the hydration solution, at a final lipid concentration of $55 \mathrm{~g} / \mathrm{l}$. The suspension of MLVs was subsequently submitted to ultrasound at $55^{\circ} \mathrm{C}$ using a $3-\mathrm{mm}$ probesonicator (ultrasonic liquid processor, Misonix Inc., Farmingdale, NY, USA). Remaining MLVs and contaminating metal from the probe were eliminated by centrifugation at $10,000 \mathrm{~g}$ for $10 \mathrm{~min}$. The resulting suspension of small unilamellar vesicles (SUVs) was then mixed with the solution of meglumine antimoniate at the final lipid to $\mathrm{Sb}$ weight ratio of 1:0.58. The mixture was immediately frozen and then dried overnight. Rehydration of the dried powder was performed at $55^{\circ} \mathrm{C}$ as follows: $20 \%$ of the original SUV volume of distilled water was added, and the mixture was vortexed and incubated for $30 \mathrm{~min}$ at $55^{\circ} \mathrm{C} ; 20 \%$ of the volume of PBS was similarly added, and the mixture was vortexed prior to the addition of $160 \%$ of the original SUV volume of PBS and incubated for $30 \mathrm{~min}$ at $55^{\circ} \mathrm{C}$. Drug-containing DRVs were separated from the free drug by centrifugation at $10,000 \mathrm{~g}$ for $30 \mathrm{~min}$ at $4^{\circ} \mathrm{C}$. The liposome pellet was then washed twice with $8 \mathrm{ml}$ PBS and finally resuspended in $2 \mathrm{ml} \mathrm{PBS}$.

The encapsulation of meglumine antimoniate into FDELs was performed as previously described (12) with the following modifications. SUVs were prepared from DSPC/ CHOL/DCP (molar ratio of 5:4:1) as described above at a final lipid concentration of $55 \mathrm{~g} / \mathrm{l}$. This suspension was frozen and subsequently dried overnight. Rehydration of the dried powder was performed with meglumine antimoniate solution as follows: $40 \%$ of the original SUV volume of meglumine antimoniate solution was added (corresponding to a lipid/Sb weight ratio of 1:0.58), and the mixture was vortexed and incubated for $30 \mathrm{~min}$ at $55^{\circ} \mathrm{C} ; 40 \%$ of the PBS volume was similarly added, and the mixture was vortexed prior to the addition of $120 \%$ of the original SUV volume of PBS and incubated for $30 \mathrm{~min}$ at $55^{\circ} \mathrm{C}$. Drug-containing FDELs were separated from the free drug by centrifugation at $10,000 \mathrm{~g}$ for $30 \mathrm{~min}$ at $4^{\circ} \mathrm{C}$. The liposome pellet was then washed twice with $8 \mathrm{ml} \mathrm{PBS}$ and finally resuspended in $2 \mathrm{ml}$ PBS.

To determine the amount of antimonial drug encapsulated in liposomes, antimony was evaluated in the liposome pellets and in the supernatant of the first centrifugation. These samples were placed in a solution of nitric acid and heated to dryness. This step was then repeated until the complete digestion of organic material. The final dry product was re-dissolved in a 1:1 mixture of concentrated hydrogen chloride and water, and boiled for $1 \mathrm{~h}$. Finally, antimony content was determined by atomic absorption spectroscopy. The encapsulation efficiency $(\% \mathrm{E})$ of meglumine antimoniate was calculated as follows: $\% \mathrm{E}=100 \mathrm{x}$ quantity of encapsulated antimony/(quantity of encapsulated antimony + quantity of unencapsulated antimony).

Young 60-80 g Golden Syrian hamsters (Mesocricetus auratus) were used to maintain the parasite and were used throughout the experiments. The Leishmania chagasi strain MHU/BR/70/BH46 was used in this study. Parasites were obtained by homogenizing fragments of liver and spleen from a freshly killed hamster which had been infected for approximately 90 days.

In order to evaluate the antileishmanial activity of the liposomal preparation, 4 groups of 10 hamsters were infected intraperitoneally with $0.1 \mathrm{ml}$ of the suspension of $L$. chagasi amastigotes (approximately $10^{8}$ para- 
sites). Seventeen days after infection, animals were treated intraperitoneally with i) meglumine antimoniate entrapped in DSPC/ CHOL/DCP FDELs (Lglu) at $60 \mathrm{mg} \mathrm{Sb} / \mathrm{kg}$, ii) free meglumine antimoniate (Glu) at 100 $\mathrm{mg} \mathrm{Sb} / \mathrm{kg}$, iii) empty liposomes (Lemp), and iv) PBS (untreated). After 55 days, animals were sacrificed, and impression smears of liver and spleen were taken. Smears were fixed with methanol, stained with $10 \%$ Giemsa (Gibco) and the number of amastigotes per 1000 host cell nuclei was counted. For each animal, about 500 host cell nuclei in the liver as well as in the spleen were evaluated for the presence of amastigotes.

Entrapment data (Table 1) indicated that the 'DRV method' is the most efficient method for the encapsulation of meglumine

Table 1 - Glucantime encapsulation into multilamellar vesicles (MLVs), dehydration-rehydration vesicles (DRVs) and freeze-dried empty liposomes (FDELS).

aN $=3 .{ }^{b} 14$ mg of lipid used.

\begin{tabular}{|c|c|c|}
\hline $\begin{array}{l}\text { Liposome } \\
\text { preparation }\end{array}$ & $\begin{array}{l}\text { Percentage of } \\
\text { entrapped } \\
\text { Glucantime } \\
\text { (mean } \pm \text { SD) }^{\mathrm{a}}\end{array}$ & $\begin{array}{c}\text { Weight ratio } \\
\text { of lipid to } \\
\text { encapsulated } \\
\mathrm{Sb}\end{array}$ \\
\hline MLVs & $12.5 \pm 1$ & 1:0.074 \\
\hline DRVs ${ }^{b}$ & $42 \pm 2$ & 1:0.25 \\
\hline FDELs ${ }^{b}$ & $31 \pm 3$ & 1:0.18 \\
\hline
\end{tabular}

antimoniate into liposomes. The 'FDEL method' gave a lower encapsulation efficiency than the DRV one, but a higher level (2.5-fold) than the conventional thin film hydration method. These results were obtained using a lipid quantity of $14 \mathrm{mg}$ and an initial lipid to $\mathrm{Sb}$ weight ratio of 1:0.58. When the lipid quantity was increased in the FDEL preparation while maintaining constant the drug to lipid ratio, an increase in encapsulation efficiency was observed. For instance, when $140 \mathrm{mg}$ of lipid was used, the encapsulation efficiency of meglumine antimoniate into FDELs reached $50 \pm 10 \%$.

Table 2 shows that treatment of Leishmania chagasi-infected hamsters with Lglu reduced parasite burdens significantly in the liver, when compared to treatment with Lemp. On the other hand, no significant difference in parasite burden was observed between the groups treated with either empty liposomes or free meglumine antimoniate (100 mg Sb/kg) and the control group. More strikingly, all animals treated with Lglu showed less than 70 amastigotes per 1000 host cell nuclei either in the liver or in the spleen, and some animals ( 4 out of 9 ) seemed to be free of parasites (Table 2). This observation is in contrast with the results obtained with the other groups in which all animals showed parasites, and at a much higher level.

The aim of the present study was to look

Table 2 - The effect of liposome-encapsulated meglumine antimoniate (Lglu, $60 \mathrm{mg} \mathrm{Sb} / \mathrm{kg}$ ), free meglumine antimoniate (Glu, $100 \mathrm{mg} \mathrm{Sb} / \mathrm{kg}$ ) and empty liposomes (Lemp) on L. chagasi parasite burdens of liver and spleen in hamsters.

$* \mathrm{P}<0.05$ compared to the Lemp group (one-way ANOVA and Tukey's test).

\begin{tabular}{|c|c|c|c|c|c|}
\hline \multirow[t]{2}{*}{ Treatment } & \multicolumn{2}{|c|}{$\begin{array}{l}\text { Mean number of } \\
\text { amatigotes/1000 host } \\
\text { cell nuclei } \pm \text { SD }\end{array}$} & \multicolumn{2}{|c|}{$\begin{array}{l}\text { Number of animals } \\
\text { with amatigotes } / 1000 \\
\text { host cell nuclei }<70 \\
\text { (total number) }\end{array}$} & \multirow[t]{2}{*}{$\begin{array}{l}\text { Number of animals } \\
\text { with no parasite } \\
\text { detected } \\
\text { (total number) }\end{array}$} \\
\hline & Liver & Spleen & Liver & Spleen & \\
\hline Lglu & $9 \pm 15^{*}$ & $13 \pm 25$ & $10(10)$ & $9(9)$ & $4(9)$ \\
\hline Glu & $1800 \pm 2000$ & $3200 \pm 4800$ & $1(11)$ & $0(10)$ & $0(11)$ \\
\hline Lemp & $2500 \pm 2600$ & $6700 \pm 9700$ & $0(8)$ & $0(7)$ & $0(8)$ \\
\hline None & $1600 \pm 1900$ & $7600 \pm 8100$ & $1(8)$ & $0(7)$ & $0(8)$ \\
\hline
\end{tabular}


for alternative methods for the encapsulation of meglumine antimoniate into liposomes, that do not suffer from the limitations of conventional ones. From the point of view of encapsulation efficiency, the methods that produce DRVs and FDELs appeared to be as efficient as the reverse-phase evaporation method that was claimed to produce encapsulation efficiency in the range of 38 to $57 \%$ (8). Moreover, comparable ratios of lipid to $\mathrm{Sb}$ were achieved.

In order to avoid stability problems during storage, such as those encountered with aqueous liposome suspensions, the DRV and FDEL preparations may be stored as intermediate lyophilized products: DRVs as a liposome-drug mixture and FDELs as preformed empty liposomes. The final rehydration step may be performed just before administration, using i) water and saline in the case of DRVs and ii) meglumine antimoniate solution in the case of FDELs. A significant advantage of the 'FDEL method' is that it does not expose the drug to lyophilization, thereby reducing the risk of chemical alteration of the drug. Moreover, the rehydration step was found to be much easier in the case of FDELs (data not shown). Finally, the FDEL preparation did show the expected high antileishmanial activity in hamsters infected with Leishmania chagasi.

Conventional therapy with meglumine antimoniate involves a multiple dosing regimen of a minimum of 28 daily intramuscular or intravenous injections of $20 \mathrm{mg} \mathrm{Sb} / \mathrm{kg}$ (13). According to pre-clinical studies in experimental animals, liposome-encapsulated meglumine antimoniate is at least 200 fold as active as the free drug (1). These data suggest that a treatment consisting of four doses of $2 \mathrm{mg} \mathrm{Sb} \mathrm{kg}^{-1}$ week ${ }^{-1}$ with meglumine antimoniate encapsulated into lipo- somes might be satisfactory, since each dose is 70-fold lower than the cumulative dose of antimony administered in one week by conventional treatment. It is noteworthy that if the preparation is used without eliminating the $50 \%$ fraction of unencapsulated drug, then the quantity of unencapsulated drug administered would represent $2 \mathrm{mg} \mathrm{Sb} \mathrm{kg}{ }^{-1}$ week $^{-1}$, which is insignificant when compared to the dose administered in the conventional treatment. This observation means that the process of preparation of DRVs and FDELs might be simplified by the omission of the last centrifugation/separation step.

Another important point that needs to be addressed is the cost of treatment with the liposomal drug when compared to conventional therapy. In a treatment using the FDEL preparation, much lower doses of antimony would be used and the cost would be determined mainly by lipids. Assuming that the cost of lipid is US\$50 per $g$ and that of meglumine antimoniate is US\$2 per $5 \mathrm{ml}$ ampoule, a four-week treatment with $2 \mathrm{mg}$ $\mathrm{Sb} \mathrm{kg}^{-1}$ week $^{-1}$ of liposomal meglumine antimoniate would cost US $\$ 120$, whereas conventional treatment $\left(20 \mathrm{mg} \mathrm{Sb} \mathrm{kg}^{-1}\right.$ day $\left.^{-1}\right)$ costs about US\$200.

In conclusion, we observed that FDELentrapped meglumine antimoniate is a suitable formulation for the treatment of visceral leishmaniasis: the preparation exhibited high antileishmanial activity, did not suffer from stability problems and was cost-effective. It is expected that treatment with this preparation would allow for a lower number of injections and a lower dose of antimony when compared to conventional treatment. Therefore, the treatment, in addition to its higher efficiency, should be safer and more comfortable. 


\section{References}

1. Alving CR (1986). Liposomes as drug carriers in leishmaniasis and malaria. Parasitology Today, 2: 101-107.

2. Croft SL (1986). Liposomes in the treatment of parasitic diseases. Pharmacy International, 7: 229-233.

3. New RRC, Chance ML \& Heath S (1981). Antileishmanial activity of amphotericin and other antifungal agents entrapped in liposomes. J ournal of Antimicrobial Chemotherapy, 8: 371-381.

4. Alving CR, Steck EA, Chapman JRWL, Waits VB, Hendricks LD, Swartz J r GM \& Hanson WL (1980). Liposomes in leishmaniasis: therapeutic effects of antimonial drugs, 8-aminoquinolines, and tetracyclines. Life Sciences, 26: 2231-2238.

5. Baillie AJ , Coombs GH, Dolan TF \& Laurie J (1986). Nonionic surfactant vesicles, niosomes, as a delivery system for the antileishmanial drug sodium stibogluco- nate. J ournal of Pharmacy and Pharmacology, 38: 502-505.

6. Storm G \& Crommelin DJ A (1998). Liposomes: Quo vadis? Pharmaceutical Science and Technology Today, 1: 19-31.

7. Alving CR \& Swartz J r GM (1984). Preparation of liposomes for use as drug carriers in the treatment of leishmaniasis. In: Gregoriadis G (Editor), Liposome Technology. Vol. II. 1st edn. CRC Press, Boca Raton, FL, 55-68.

8. Rao LS (1986). Anti-Leishmanial Pharmaceutical Formulation. US Patent Number: $4,594,241$.

9. Williams DM, Carter $K C \&$ Baillie $A J$ (1995). Visceral leishmaniasis in BALB/C mouse: a comparison of the in vivo activity of five non-ionic surfactant vesicle preparations of sodium stibogluconate. J ournal of Drug Targeting, 3: 1-7.

10. Roberts WL, McMurray WJ \& Rainey PM
(1998). Characterization of the antimonial antileishmanial agent meglumine antimonate (Glucantime). Antimicrobial Agents and Chemotherapy, 42: 1076-1082.

11. Kirby C \& Gregoriadis G (1984). Dehydration-rehydration vesicles: a simple method for high yield drug entrapment in liposomes. Biotechnology, 2: 979-984.

12. Yachi K, Harashima H, Kikuchi H, Sudo R, Yamauchi $\mathrm{H}$, Ebihara $\mathrm{K}$, Matsuo $\mathrm{H}$, Funato K \& Kiwada H (1996). Biopharmaceutical evaluation of the liposomes prepared by rehydration of freeze-dried empty liposomes (FDELs) with an aqueous solution of a drug. Biopharmaceutics and Drug Disposition, 17: 589-605.

13. Berman J D (1997). Human leishmaniasis: clinical, diagnostic and chemotherapeutic developments in the last 10 years. Clinical Infectious Diseases, 24: 684-703. 